\title{
Hypoxia-Inducible Factor-1
}

National Cancer Institute

\section{Source}

National Cancer Institute. Hypoxia-Inducible Factor-1. NCI Thesaurus. Code C17690.

Hypoxia-inducible factor-1 (HIF1) is a transcription factor found in mammalian cells

cultured under reduced oxygen tension that plays an essential role in cellular and

systemic homeostatic responses to hypoxia. HIF1 is a heterodimer composed of a 120-

kD HIF1-alpha subunit complexed with a 91- to 94-kD HIF1-beta subunit. 